\title{
REGULARITY PROPERTIES OF A CERTAIN CLASS OF SURFACES
}

\section{H. BUSEMANN AND W. FELLER}

Introduction. Convex curves in the plane and convex surfaces in space have many at first sight unexpected regularity properties. ${ }^{1}$ The stringency of the convexity condition makes it desirable to find a geometric property of convex curves or surfaces which has similar analytical implications but applies to a wider class of geometrical objects. The first and most studied generalization of convexity is finiteness of the order: a curve or surface is said to be of order $n$ if its intersection with any straight line consists of at most $n$ connected sets $^{2}$ (segments, points, or rays). However, this condition proves to be much too weak. ${ }^{3}$ On the other hand, the dual condition, finiteness of class, will turn out to be more restrictive than necessary.

The present paper will show that for curves finiteness of the total curvature is an entirely satisfactory condition. It includes in particular all curves of finite class. More generally, hypersurfaces in $E^{n}$ with finite total curvature seem to share the most important differentiability properties with convex hypersurfaces.

The present results on curves with finite total curvature and the modern theory of functions of a real variable lead to the following surprising fact concerning surfaces in $E^{3}$ : If the paratingens ${ }^{4}$ of $\Phi$ at a given point p leaves out at least one line $L$, and if, locally, the plane sections of $\Phi$ parallel to $L$ have uniformly bounded total curvature, then the following facts hold simultaneously at almost all points $q$ of $\Phi$ : the surface $\Phi$ has a tangent plane $\Pi$ at $q$. All plane sections $(\neq \Pi)$ of $\Phi$ through $p$ have a curvature ${ }^{5}$ at $p$, and these curvatures satisfy the theorems of Meusnier and Euler.

Received by the editors April 13, 1945.

${ }^{1}$ For curves compare Jessen [1], for surfaces Busemann-Feller [1], and for hypersurfaces Alexandrov [1]. Numbers in brackets refer to the references cited at the end of the paper.

2 This formulation is due to Hjelmslev [2] and has the obvious advantage not to exclude polygons, polyhedrons, ruled surfaces, $\cdots$.

${ }^{8}$ Compare Marchaud [1], Haupt [1], and $\$ 2$ of the present paper.

4 This concept is due to Bouligand, see Bouligand [1]. The paratingens of $\Phi$ represented parametrically by $p(u, v)$ at $\left(u_{0}, v_{0}\right)$ consists of all non-oriented lines $G$ which are limits of sequences of non-oriented lines $G\left(p\left(u_{\nu}^{\prime}, v_{\nu}^{\prime}\right), p\left(u_{\nu}, v_{\nu}\right)\right)$ with $\left(u_{\nu}^{\prime}, v_{\nu}^{\prime}\right)$ $\neq\left(u_{\nu}, v_{\nu}\right)$ and $u_{\nu}^{\prime}, u_{\nu} \rightarrow u_{0}, v_{\nu}^{\prime}, v_{\nu} \rightarrow v_{0}$. For the definition of total curvature see the following $\$ 1$.

8 More precisely the ordinary curvature as defined at the end of $\$ 1$. 
It will be seen that the hypotheses can be somewhat reduced. Theorems (4.5) and (4.8) are believed to be of interest for the theory of real variables.

1. Curves of finite total curvature. For $a \leqq t \leqq b$ let the point $p(t)=(x(t), y(t))$ of the $(x, y)$-plane describe a continuous curve $C$ which is locally a Jordan arc: that is to say, for every $t_{0}$ let there be an $\eta\left(t_{0}\right)>0$ such that the mapping $p(t) \rightarrow t$ is topological for $\left|t-t_{0}\right| \leqq \eta\left(t_{0}\right)$, or $a \leqq t \leqq a+\eta(a)$, or $b-\eta(b) \leqq t \leqq b,{ }^{6}$ respectively.

The oriented straight line $G$ is called a paratangent of $C$ at $t_{0}$ if there exist sequences $t_{k}^{\prime} \rightarrow t_{0}, t_{k}^{\prime \prime} \rightarrow t_{0}$ with $t_{k}^{\prime}<t_{k}^{\prime \prime}$ and such that the oriented line $G\left(p\left(t_{k}^{\prime}\right), p\left(t_{k}^{\prime \prime}\right)\right) \equiv G\left(t_{k}^{\prime}, t_{k}^{\prime \prime}\right)$ tends to $G$. If, in particular, the two sequences can be chosen so that $t_{k}^{\prime} \leqq t_{0} \leqq t_{k}^{\prime \prime}$, then the paratangent $G$ is called a tangent of $C$ at $t_{0}$. If $t_{k}^{\prime \prime} \leqq t_{0}\left(t_{k}^{\prime \prime}=t_{0}\right)$, then $G$ is called a left-hand paratangent (tangent) of $C$ at $t_{0}$; right-hand tangents and paratangents are defined similarly. ${ }^{7}$

(1.1) The paratangent (tangent) of $C$ at $t_{0}$ is unique if and only if the one-sided paratangents (tangents) of $C$ at $t_{0}$ are unique and coincide.

The statement is obvious, but it should be noticed that it holds only for oriented tangents and paratangents.

The mean value theorem holds for these tangents in the following form :

(1.2) If $t_{1}<t_{2}$ and $p\left(t_{1}\right) \neq p\left(t_{2}\right)$, then a value $t_{0}$ between $t_{1}$ and $t_{2}$ exists such that a suitable tangent of $C$ at $t_{0}$ is parallel either to $G^{+}=G\left(t_{1}, t_{2}\right)$ or to its opposite orientation $G^{-} .^{8}$

A loop joining two points (for example, a strophoid) will show that a tangent parallel to $G^{+}$need not exist. The statement (1.2) is easily proved by considering the points of $C$ for which the distance from $G^{+}$ is maximal.

For every $\phi, 0 \leqq \phi<2 \pi$, define $N(\phi)$ (possibly $N(\phi)=\infty$ ) as the number of values $t$ for which a tangent of $C$ at $t$ forms the angle $\phi$ with the positive $x$-axis. If $N(\phi)$ is summable, $\kappa=\int_{0}^{2 x} N(\phi) d \phi$ is called the total curvature of $C$, otherwise put $\kappa=\infty$.

If the tangent $T(t)$ of $C$ is unique for every $t$ and varies continuously with $t$, then the end point of the unit vector with origin $(0,0)$ and

- The end points will frequently require similar obvious changes, but these will in general not be mentioned.

' One-sided tangents or paratangents will be used as common word for righthand and left-hand tangents or paratangents.

8 For curves given in the form $y=f(x)$ this result (compare (3.1)) is due to Hjelmslev [1]. 
parallel to $T(t)$ traverses a continuous curve on the unit circle whose (finite or infinite) length equals $k .{ }^{9}$ This justifies the above nomenclature. It will now be shown that curves of finite total curvature have an important property of convex curves.

(1.3) ThEOREM. A curve of finite total curvature has everywhere unique one-sided paratangents.

Suppose that there exist two left-hand paratangents $T^{\prime}$ and $T^{\prime \prime}$ at $t_{0}$. Then at least one of the two angular intervals between $T^{\prime}$ and $T^{\prime \prime}$ has the property that to every line $L$ contained in it there exist infinitely many chords of $p(t)$ parallel to $L$. By the mean value theorem there exist therefore infinitely many points $t_{\nu} \rightarrow t$ such that a tangent at $t_{\nu}$ is parallel or opposite to $L$. Therefore, for every $\phi$ of one of the two angular intervals between $T^{\prime}$ and $T^{\prime \prime}$ either $N(\phi)=\infty$ or $N(-\phi)=\infty$ so that $N(\phi)$ can not be finite almost everywhere.

For any curve $C$ let $t_{0}$ be a value for which the one-sided paratangents are unique. Then it is easily seen that the paratingens of $C$ at $t_{0}$, that is, the set of all paratangents at $t_{0}$, is determined as follows: Let $T_{1}$ and $T_{2}$ be the two one-sided paratangents, $T_{1}^{\prime}$ and $T_{2}^{\prime}$ their positive subrays with origin $p\left(t_{0}\right)$. Then either the angle between $T_{1}^{\prime}$ and $T_{2}^{\prime}$ has measure $\pi$, in which case the paratingens is the whole plane and we say that $C$ has at $t_{0}$ a cusp; or $T_{1}^{\prime}$ and $T_{2}^{\prime}$ bound exactly one angle of measure $\omega\left(t_{0}\right)<\pi$. This closed angle together with its vertical angle form the paratingens of $C$ at $t_{0}$ whose measure is defined as $\omega\left(t_{0}\right)$.

The importance of the last theorem is shown by the following simple facts :

(1.4) If the curve $C: p(t), a \leqq t \leqq b$, has everywhere unique one-sided paratangents then:

(a) Every paratangent of $C$ is a tangent.

(b) If $t_{v} \rightarrow t_{0}-0$ and $T_{\nu}$ is a tangent of $C$ at $t_{v}$, then $T_{\nu}$ tends to the lefthand tangent of $C$ at $t_{0}$.

(b') If $t_{\nu} \rightarrow t_{0}$, and the tangent $T$ of $C$ at $t_{0}$ is unique, then $T_{\nu} \rightarrow T$.

(c) For every $\eta>0$ the set of those values $t$ for which $\omega\left(t_{0}\right)>\eta$ is finite.

(c') The number of t's for which the paratangent of $C$ is not unique is at most countable.

To see (a) let $t_{\nu}^{\prime}<t_{\nu}^{\prime \prime}$ and $t_{\nu}^{\prime} \rightarrow t_{0}, t_{\nu}^{\prime \prime} \rightarrow t_{0}, G\left(t_{\nu}^{\prime}, t_{\nu}^{\prime \prime}\right) \rightarrow G$. If $t_{\nu}^{\prime} \leqq t_{0} \leqq t_{\nu}^{\prime \prime}$ for infinitely many $\nu$ nothing is to be proved. If $t_{\nu}^{\prime} \leqq t_{\nu}^{\prime \prime} \leqq t_{0}$, then

\footnotetext{
- This is a geometric formulation of a known theorem in the theory of real variables; compare, for example, Saks [1].
} 
$G\left(t_{\nu}^{\prime}, t_{\nu}^{\prime \prime}\right)$ tend by hypothesis to the same limit as $G\left(t_{\nu}^{\prime}, t_{0}\right)$, so that $C$ is a tangent.

To prove (b) choose $t_{\nu}^{\prime}$ and $t_{\nu}^{\prime \prime}$ with $t_{\nu}^{\prime} \leqq t_{\nu} \leqq t_{\nu}^{\prime \prime}<t_{0}$ such that $0<t_{\nu}^{\prime \prime}-t_{\nu}^{\prime}<1 / \nu$ and the smaller angle between $G\left(t_{\nu}^{\prime}, t_{\nu}^{\prime \prime}\right)$ and $T_{\nu}$ has measure less than $1 / \nu$. Then $t_{\nu}^{\prime} \rightarrow t_{0}$ and $G\left(t_{\nu}^{\prime}, t_{\nu}^{\prime \prime}\right)$ tends by (a) to the left-hand tangent of $C$ at $t_{0}$.

If (c) were not true, then a sequence $t_{v}$ with $\omega\left(t_{v}\right)>\eta$ would exist. It may be assumed that $t_{\nu} \rightarrow t_{0}-0$. The two one-sided tangents of $C$

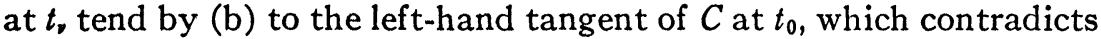
the hypothesis $\omega\left(t_{\nu}\right)>\eta$. If $C$ has the finite total curvature $\kappa$, the statement $\left(\mathrm{c}^{\prime}\right)$ may be improved to $\sum \omega\left(t_{\nu}\right) \leqq \kappa$, where the $t_{\nu}$ are the values of $t$ where the paratangent is not unique. In particular, there are fewer than $\alpha / \pi$ cusps.

The uniqueness of the one-sided paratangents allows us also to associate with every tangent $T$ of $C$ at $t$ an angle $\psi(t)$ between the tangent and the positive $x$-axis $(\psi(t)$ not necessarily between 0 and $2 \pi$ ) which, although multivalued, varies with $t$ in very much the same manner as when $C$ has a continuous tangent; let $\tau_{1}<\tau_{2}<\ldots<\tau_{r}$ denote the cusps of $C$; and put $a=\tau_{0}, b=\tau_{r+1}$. Then $\tau_{0}<\tau_{1}, \tau_{r}<\tau_{r+1}$. Consider a subarc $C_{i}: \tau_{i} \leqq t \leqq \tau_{i+1}$ of $C$. Define as tangents of $C_{i}$ at $\tau_{i}$ and $\tau_{i+1}$ the right-hand and left-hand tangents of $C$ at $\tau_{i}$ and $\tau_{i+1}$, respectively. By (1.4b) there is for every $\tau, \tau_{i} \leqq \tau \leqq \tau_{i+1}$, a $\delta(\tau)>0$ such that the smaller angle between any two paratangents of $C$ for $|\tau-t|<\delta(\tau)\left(0 \leqq \tau-\tau_{i}<\delta\left(\tau_{i}\right), 0 \leqq \tau_{i+1}-\tau<\delta\left(\tau_{i+1}\right)\right)$ is less than $\pi$. A finite number of these intervals belonging to values $t_{1}=\tau_{i}<t_{2}<\ldots$ $<t_{m}=\tau_{i+1}$ will cover the interval $\left(\tau_{i}, \tau_{i+1}\right)$ so that only consecutive intervals $\left|t_{i}-t\right|<\delta\left(\tau_{i}\right)$ have common points. If we fix the value $\psi\left(\tau_{i}\right)=\psi\left(t_{1}\right)$ arbitrarily, then the angle $\psi(\tau)$ belonging to all $t$ in $\left|t_{1}-t\right|<\delta\left(\tau_{i}\right)$ is uniquely determined by the requirement that $\left|\psi(t)-\psi\left(\tau_{i}\right)\right|<\pi$. The same requirement and the already defined value of $\psi(t)$ for common points of $\left|t_{i}-t\right|<\delta\left(t_{i}\right), i=1,2$, determine $\psi(t)$ uniquely for $\left|t_{2}-t\right|<\delta\left(t_{2}\right)$. Continuing in the same way one defines $\psi(t)$ for all $t$ on $C_{i}$. Notice that $\psi\left(\tau_{i}+0\right)=\psi\left(\tau_{i}\right)$ and $\psi\left(\tau_{i+1}-0\right)$ $=\psi\left(\tau_{i+1}\right)$.

Now choose $\psi(a)$ so that $0 \leqq \psi(a)<2 \pi$. Then the angles $\psi(t)$ are determined for $a \leqq t \leqq \tau_{1}$. As values $\psi\left(\tau_{1}\right)$ take those with $\psi\left(\tau_{1}-0\right) \leqq \psi\left(\tau_{1}\right)$ $\leqq \psi\left(\tau_{1}-0\right)+\pi$. As initial value on $C_{1}$ choose $\psi\left(\tau_{1}-0\right)+\pi$, then $\psi(t)$ is determined on $C_{2}$, and so forth. A consequence of this determination of $\psi(t)$ is that for any $t$ the limits $\psi(t-0)$ and $\psi(t+0)$ exist and that for any $\psi(t)$ belonging to $t$ either

(1.5) $\psi(t-0) \leqq \psi(t) \leqq \psi(t+0)$ or $\psi(t-0) \geqq \psi(t) \geqq \psi(t+0)$. 
(1.6) If $C$ has total curvature $\kappa$, then for any $a=t_{1}<t_{2}<\cdots<t_{n}=b$ and any choice of the values $\psi\left(t_{i}\right)$

For by (1.5)

$$
\sum_{r=1}^{n-1}\left|\psi\left(t_{i+1}\right)-\psi\left(t_{i}\right)\right| \leqq \kappa
$$

$$
\begin{aligned}
\sum\left|\psi\left(t_{i}\right)-\psi\left(t_{i+1}\right)\right| \leqq & \sum\left|\psi\left(t_{i}+0\right)-\psi\left(t_{i+1}-0\right)\right| \\
& +\sum\left|\psi\left(t_{i}+0\right)-\psi\left(t_{i}-0\right)\right| .
\end{aligned}
$$

Now $\psi(t)$ takes on every value between $\psi\left(t_{i}+0\right)$ and $\psi\left(t_{i+1}-0\right)$ at least once in $\left(t_{i}, t_{i+1}\right)$ and every value between $\psi\left(t_{i}-0\right)$ and $\psi\left(t_{i}+0\right)$ exactly once at $t_{i}$, which proves (1.6).

Let $C_{i}, \tau_{i} \leqq t \leqq \tau_{i+1}$, denote the same subarc of $C$ as above. For every $\tau_{0}$ the paratingens of $C_{i}$ at $t_{0}$ leaves out at least one line $L^{\prime}$. Choose the perpendicular $L$ to $L^{\prime}$ as an $x^{\prime}$-axis and $L^{\prime}$ as a $y^{\prime}$-axis. Then for a suitable $\delta>0$, the subarc $\left|t_{0}-t\right| \leqq \delta$ has a representation $y^{\prime}=f\left(x^{\prime}\right), \quad-\delta_{1} \leqq x^{\prime} \leqq \delta_{2}, \quad \delta_{i}>0$, and the difference quotients $\left(f\left(x_{1}^{\prime}\right)-f\left(x_{2}^{\prime}\right)\right) /\left(x_{1}^{\prime}-x_{2}^{\prime}\right)$ are bounded ${ }^{10}$ in $\left(-\delta_{1}, \delta_{2}\right)$, since the paratingens of $C$ at $t_{0}$ is a closed set. Therefore the arc $\left|t_{0}-t\right| \leqq \delta$ of $C$ has the finite length $\int_{-\delta_{1}}^{\delta_{2}}\left\{1+f^{\prime 2}\left(x^{\prime}\right)\right\}^{1 / 2} d x^{\prime}$. A trivial application of the Heine-Borel theorem yields that $C_{i}$ has finite length, so that $C$ is also rectifiable. Introduce the arc length $s$ as parameter on $C$, and let $\psi(t)$ become the function $\phi(s)$. Let $\phi^{*}(s)$ denote any of the values $\phi(s)$. Then $\phi^{*}(s)$ has by (1.6) bounded variation so that $\phi^{* \prime}(s)$ exists for almost all $s$. If $\phi(s)$ denotes any other value of $\phi(s)$, then because of $(1.5), \Phi^{\prime}(s)$ exists and equals $\phi^{* \prime}(s)$ whenever $\phi^{* \prime}(s)$ exists. Therefore we shall simply speak of $\phi^{\prime}(s)$. Whenever $\phi^{\prime}(s)$ exists, the tangent $T(s)$ of $C$ is unique.

The geometric equivalent to the existence of $\phi^{\prime}(s)$ is this : Let $\nu(s)$ be the normal to $T(s)$ at $p(s)$, and denote by $\nu(s+h)$ the normal to any tangent of $C$ at $(s+h)$, and by $d_{s}(h)$ the distance (possibly $\infty$ ) of the intersection of $\nu(s)$ and $\nu(s+h)$ from $p(s)$. Then $1 / d_{s}(h) \rightarrow\left|\phi^{\prime}(s)\right|$, and $\left|\phi^{\prime}(s)\right|$ is the so-called strong curvature of $C$ at $s$.

(1.7) A curve of finite total curvature has almost everywhere (with respect to the arclength $s$ ) a finite strong curvature.

The (ordinary) curvature of $C$ at a point $s$ where $C$ has a unique tangent $T(s)$ is defined as follows: Let $r_{s}(h)$ denote the radius (possibly $\infty)$ of the circle which has tangent $T(s)$ at $p(s)$ and passes through $p(s+h)$. If $1 / r_{s}(h)$ converges, then the limit is the curvature

\footnotetext{
${ }^{10}$ Compare Bouligand [1, p. 123].
} 
of $C$ at s. For any curve the existence of the strong curvature $\lim \left(1 / d_{s}(h)\right)$ implies the existence of the curvature $\lim \left(1 / r_{s}(h)\right)$; for convex curves the converse holds. ${ }^{11}$ This is not necessarily true for curves of finite total curvature.

2. Examples. Before passing on to surfaces, we exhibit three examples of curves. (a) We show that curves of finite total curvature are really essentially more general than convex curves. This will be accomplished by constructing a curve with $N(\phi) \leqq 3$ which is not the union of a countable set of convex arcs and a set of measure 0 . (b) We prove the statement at the end of $\$ 1$. (c) We show that finiteness of the order is not an adequate generalization of convexity.

Let $\kappa_{1}, \kappa_{2}, \cdots$ be any finite or countable number of closed disjoint subarcs of a semicircle $\kappa$. Denote by $\kappa_{\nu}^{\prime}$ the image of $\kappa_{\nu}$ under the reflection in the line $G\left(a_{\nu}, b_{\nu}\right)$ connecting the end points $a_{\nu}, b_{\nu}$ of $\kappa_{\nu}$, and replace $\kappa_{\nu}$ by $\kappa_{\nu}^{\prime}$ for every $\nu$. The curve $C^{\prime}$ thus obtained from $\kappa$ has $N(\phi) \leqq 3$. If the $\kappa_{v}$ are chosen so that the set $\kappa-\sum \kappa_{\nu}$ is a nowhere dense subset of $\kappa$ with positive measure, $C^{\prime}$ will not be the union of a countable number of convex arcs and a set of measure 0 .

It requires a little more to find an example for (b). With the same notation as before but arbitrary $\kappa_{\nu}$ take a subarc $\kappa_{\nu}^{*}$ of $\kappa_{\nu}^{\prime}$ whose end points $a_{\nu}^{\prime}$ and $b_{\nu}^{\prime}$ are close to but different from $a_{\nu}$ and $b_{\nu}$, respectively, and replace $\kappa_{\nu}^{*}$ by its image $\kappa_{\nu}^{\prime \prime}$ under reflection in $G\left(a_{\nu}^{\prime}, b_{\nu}^{\prime}\right)$. For the curve $C^{\prime \prime}$ thus obtained from $C^{\prime}$ the function $N(\phi)$ is at most 6 .

Now let $\kappa$ be the semicircle $x \geqq 0$ of the circle $x^{2}+y^{2}=1$, and $E$ any curve which passes through $(0,1)$ and lies except for $(0,1)$ entirely in the unit circle and has there $y=1$ as tangent, and curvature 1 . Then any curve between $\kappa$ and $E$ through $(0,1)$ will have curvature 1 at $(0,1)$. Now choose $a_{p}=\left(\cos \left(\pi / 2^{2 \nu-1}\right)\right.$, $\left.\sin \left(\pi / 2^{2 \nu-1}\right)\right)$, $b_{\nu}=\left(\cos \left(\pi / 2^{2 v}\right)\right.$, sin $\left.\left(\pi / 2^{2 \nu}\right)\right)$ and $a_{\nu}^{\prime}$ and $b_{\nu}^{\prime}$ so close to $a_{\nu}$ and $b_{\nu}$ that the arc $\kappa_{v}^{\prime \prime}$ lies between $\kappa$ and $E$. Then $C^{\prime \prime}$ has curvature 1 at $(0,1)$. But it does not have a strong curvature at $(0,1)$; for a parallel $P_{\nu}$ through $a_{\nu}$ to the radius of $\kappa$ from $(0,0)$ to $b_{\nu}$ is a normal to $C^{\prime \prime}$ at $a_{\nu}$. The equation of $P_{\nu}$ is $y-\sin \left(\pi / 2^{2 \nu-1}\right)=\tan \left(\pi / 2^{2 \nu}\right)\left(x-\cos \left(\pi / 2^{2 \nu-1}\right)\right)$, hence $P_{v}$ intersects the $x$-axis, which is the normal to $C^{\prime \prime}$ at $(0,1)$, at

$$
x_{\nu}=\cos \left(\pi / 2^{2 p-1}\right)-\frac{\sin \left(\pi / 2^{2 p-1}\right)}{\tan \left(\pi / 2^{2 \nu}\right)} \rightarrow-1 .
$$

Curves of order 3 are the union of at most 4 convex $\operatorname{arcs}^{12}$ and have therefore all differentiability properties of convex curves.

"11 See Jessen [1].

${ }^{12}$ See Marchaud [1, p. 88, Thtorème IV]. 
Haupt ${ }^{18}$ constructed an example of a curve of order 4 , where at a set $\mu$ of positive measure the paratingens is the whole plane. The strong curvature can, of course, not exist at any point of $\mu$. An example of a curve of order at most 8 with the same properties can easily be constructed as follows: With the above notations choose the $\kappa_{\nu}$ again so that $\kappa-\sum \kappa_{\nu}$ is nowhere dense and has positive measure. Construct a semicircle $\bar{\kappa}_{\nu}$ with two suitably chosen points $c_{\nu}, d_{\nu}$ of $\kappa_{\nu}$ as end points such that $\bar{\kappa}_{\nu}$ is contained in the circular segment $S_{\nu}$ bounded by $\kappa_{\nu}$ and the chord from $a_{\nu}$ to $b_{\nu}$. Let $\bar{C}$ be the curve obtained from $\kappa$ by replacing the arc from $c_{\nu}$ to $d_{\nu}$ by $\bar{\kappa}_{\nu}$. Since any straight line intersects at most two segments $S_{\nu}$, and has at most 4 common points with the subarc of $\bar{C}$ in $S_{\nu}$, it follows that $\bar{C}$ has at most order 8 (actually it has order 6 ). The paratingens is the whole plane at every point of $\kappa-\sum \kappa_{\nu}$. If the corners of $\bar{C}$ at $c_{\nu}$ and $d_{\nu}$ are smoothed off by changes taking place in a sufficiently small neighborhood of $c_{\nu}$ and $d_{\nu}$, a curve $\bar{C}^{\prime}$ is obtained, where the tangent exists and is continuous, hence the paratingens is everywhere a straight line, but the strong curvature exists at no point of $\kappa-\sum \kappa_{\nu}$. The question whether a curve of finite order has almost everywhere an ordinary curvature is open.

3. Auxiliary facts on real variables. The results of $\$ 1$ can be applied to surfaces $\Phi: p(u, v)=(x(u, v), y(u, v), z(u, v)),-\infty<u, v<\infty$, in a Cartesian $(x, y, z)$-space with the following properties:

I. For every pair $\left(u_{0}, v_{0}\right)$ there is $a \delta\left(u_{0}, v_{0}\right)>0$ so that the mapping $p(u, v) \rightarrow(u, v)$ is topological for $\left|u-u_{0}\right|<\delta$ and $\left|v-v_{0}\right|<\delta$.

II. For every $\left(u_{0}, v_{0}\right)$ there is at least one line $L$ not contained in the paratingens $^{14}$ of $\Phi$ at $\left(u_{0}, v_{0}\right)$.

III. There is a positive $\delta^{\prime} \leqq \delta$ such that planes parallel to $L$ intersect the part $\left|u-u_{0}\right| \leqq \delta^{\prime},\left|v-v_{0}\right| \leqq \delta^{\prime}$ of $\Phi$ in curves of uniformly bounded total curvature.

Some observations to explain the content of conditions II and III are in order:

There is a positive $\delta^{*} \leqq \delta$ such that with $L$ as $z^{\prime}$-axis and the plane II perpendicular to $L$ as $\left(x^{\prime}, y^{\prime}\right)$-plane the part $\left|u-u_{0}\right|<\delta^{*},\left|v-v_{0}\right|<\delta^{*}$ of $\Phi$ is represented in the form $z^{\prime}=f\left(x^{\prime}, y^{\prime}\right)$, where $\left(x^{\prime}, y^{\prime}\right)$ traverses some open set $\Gamma$ in $\Pi$ containing $x^{\prime}=0, y^{\prime}=0$. Clearly the number $\delta^{*}$ may in addition be chosen so that the difference quotients are bounded, that is,

$$
\left|f\left(x_{1}^{\prime}, y_{1}^{\prime}\right)-f\left(x_{2}^{\prime}, y_{2}^{\prime}\right)\right| /\left\{\left(x_{1}^{\prime}-x_{2}^{\prime}\right)^{2}+\left(y_{1}^{\prime}-y_{2}^{\prime}\right)^{2}\right\}^{1 / 2}<M
$$

${ }^{13}$ See Haupt [1].

${ }^{14}$ See footnote 4. 
for $\left(x_{i}^{\prime}, y_{i}^{\prime}\right) \in \Gamma$. The planes parallel to $L$ intersect therefore for $\delta^{\prime}<\delta^{*}$ the part $\left|u-u_{0}\right| \leqq \delta^{\prime},\left|v-v_{0}\right| \leqq \delta^{\prime}$ of $\Phi$ in Jordan arcs of the form $p(u(t), v(t))$ so that the concept of total curvature becomes applicable. The set $\left|u-u_{0}\right|<\delta^{\prime},\left|v-v_{0}\right|<\delta^{\prime}$ corresponds to an open set $\Gamma^{\prime}$ in $\Pi$ which contains $x^{\prime}=0, y^{\prime}=0$. Therefore an $\eta>0$ exists such that the domain $\left|x^{\prime}\right|<\eta,\left|y^{\prime}\right|<\eta$ is contained in $\Gamma^{\prime}$; it corresponds to some open set $\Delta$ containing $\left(u_{0}, v_{0}\right)$ in the $(u, v)$-plane.

This leads to a function $f\left(x^{\prime}, y^{\prime}\right)$ with the following properties:

(a) $f\left(x^{\prime}, y^{\prime}\right)$ is defined and continuous for $\left|x^{\prime}\right| \leqq \eta,\left|y^{\prime}\right| \leqq \eta$.

(b) $\left|f\left(x_{1}^{\prime}, y_{1}^{\prime}\right)-f\left(x_{2}^{\prime}, y_{2}^{\prime}\right)\right| /\left\{\left(x_{1}^{\prime}-x_{2}^{\prime}\right)^{2}+\left(y_{1}^{\prime}-y_{2}^{\prime}\right)^{2}\right\}^{1 / 2}<M<\infty$.

(c) All sections $a x^{\prime}+b y^{\prime}+c=0$ with $z^{\prime}=f\left(x^{\prime}, y^{\prime}\right)$ have total curvature less than $k<\infty$.

Before proceeding to prove the result mentioned in the introduction, some facts on functions of a real variable will be recalled. Let $f(x)$ be defined and continuous for $a \leqq x \leqq b$. The mean value theorem (1.2) becomes then : 9

(3.1) If $a \leqq x_{1}<x_{2} \leqq b$ then an $x_{0}$ between $x_{1}$ and $x_{2}$ and a tangent of $y=f(x)$ (tangent in the above sense) at $x_{0}$ exist whose slope $f^{*}\left(x_{0}\right)$ satisfies the relation $f^{*}\left(x_{0}\right)=\left\{f\left(x_{2}\right)-f\left(x_{1}\right)\right\} /\left\{x_{2}-x_{1}\right\}$.

The first and second Schwarz derivatives $D^{\prime} f\left(x_{0}\right)$ and $D^{\prime \prime} f\left(x_{0}\right)$ of $f$ at $x_{0}$ are defined by

$$
\begin{aligned}
D^{\prime} f\left(x_{0}\right) & =\lim _{h \rightarrow 0} \frac{f\left(x_{0}+h\right)-f\left(x_{0}-h\right)}{2 h}, \\
D^{\prime \prime} f\left(x_{0}\right) & =\lim _{h \rightarrow 0} \frac{f\left(x_{0}+h\right)+f\left(x_{0}-h\right)-2 f\left(x_{0}\right)}{h^{2}} .
\end{aligned}
$$

If $f^{\prime}\left(x_{0}\right)$ exists, then $D^{\prime} f\left(x_{0}\right)$ exists, but not conversely. However, ${ }^{15}$ if $D^{\prime} f\left(x_{0}\right)$ exists in a set of positive measure $E$ then $f^{\prime}(x)$ exists almost everywhere (a.e.) in $E$.

Let the difference quotients of $f(x)$ be bounded by $M$ and let the total curvature of $y=f(x)$ be at most $\kappa$. If $f^{*}(x)$ denotes the slope of any tangent of $y=f(x)$ at $x$, it follows from $\S 1$ that $f^{*}(x)$ has variation $V \leqq \kappa\left(1+M^{2}\right)^{1 / 2}$. The derivative $f^{\prime}(x)$ exists a.e. in $(\mathrm{a}, \mathrm{b})$. At all points where $f^{\prime}(x)$ exists, the existence of $f^{* \prime}(x)$ for one function $f^{*}(x)$ implies the existence of the derivative for every other slope function, and the derivatives are equal; they will be denoted by $f^{\prime \prime}(x)$.

1s This is a special case of the general and deeper theorem of Marcinkiewicz and Zygmund [1] that the existence of the $k$ th Riemann derivative of $f(x)$ in a set $E$ of positive measure implies the existence a.e. in $E$ of the $k$ th de la Valee Poussin derivative, because the $k$ th Riemann derivative coincides for $k=1,2$, with the Schwarz derivative. 
If $f^{\prime \prime}(x)$ exists then $D^{\prime \prime} f\left(x_{0}\right)$ and the so-called second de la Vallee Poussin derivative

$$
d^{\prime \prime} f\left(x_{0}\right)=\lim \frac{2}{h}\left\{\frac{f\left(x_{0}+h\right)-f\left(x_{0}\right)}{h}-f^{\prime}\left(x_{0}\right)\right\}
$$

exist and equal $f^{\prime \prime}\left(x_{0}\right)$. The existence of $f^{\prime \prime}\left(x_{0}\right)$ and $d^{\prime \prime} f\left(x_{0}\right)$ are equivalent to the existence of the strong and ordinary curvatures of $y=f(x)$ at $x_{0}{ }^{16}$ respectively.

$d^{\prime \prime} f(x)$ may exist in a set $E$ of positive measure, without $f^{\prime \prime}(x)$ existing anywhere in $E .{ }^{17}$ If $d^{\prime \prime} f\left(x_{0}\right)$ exists, then $D^{\prime \prime} f\left(x_{0}\right)$ exists but not conversely, however $:^{15}$

(3.2) If $D^{\prime \prime} f(x)$ exists in a set $E$ of positive measure, then $d^{\prime \prime} f(x)$ exists a.e. in $E$.

If $f(x, y)$ is defined and continuous for $|x| \leqq \eta,|y| \leqq \eta$ all these concepts can be applied to derivatives of $f(x, y)$ in a given direction $\alpha$ and lead to concepts like $f_{\alpha}^{*}\left(x_{0}, y_{0}\right), f_{\alpha}^{\prime}\left(x_{0}, y_{0}\right), D_{\alpha}^{\prime} f\left(x_{0}, y_{0}\right), f_{\alpha}^{\prime \prime}\left(x_{0}, y_{0}\right)$, $D_{\alpha}^{\prime \prime} f\left(x_{0}, y_{0}\right)$ and $d_{\alpha}^{\prime \prime} f\left(x_{0}, y_{0}\right)$, where, for example,

$$
\begin{aligned}
D_{\alpha}^{\prime} f\left(x_{0}, y_{0}\right) & =\lim \frac{1}{2 h}\left\{f\left(x_{0}+h \cdot \cos \alpha, y_{0}+h \cdot \sin \alpha\right)\right. \\
\left.-f\left(x_{0}-h \cdot \cos \alpha, y_{0}-h \cdot \sin \alpha\right)\right\}, & \\
d_{\alpha}^{\prime} f\left(x_{0}, y_{0}\right) & =\lim \frac{2}{h}\left\{\frac{f\left(x_{0}+h \cdot \cos \alpha, y_{0}+h \cdot \sin \alpha\right)-f\left(x_{0}, y_{0}\right)}{h}-f_{\alpha}^{\prime}\left(x_{0}, y_{0}\right)\right\} .
\end{aligned}
$$

For fixed $\alpha$ the set where any one of these derivatives exists is measurable.

For any interval $I: x^{\prime}<x<x^{\prime \prime}, y^{\prime}<y<y^{\prime \prime}$ put

$$
\Delta(I, f)=f\left(x^{\prime}, y^{\prime}\right)+f\left(x^{\prime \prime}, y^{\prime \prime}\right)-f\left(x^{\prime}, y^{\prime \prime}\right)-f\left(x^{\prime \prime}, y^{\prime}\right) .
$$

The variation of $f$ in the rectangle $S:|x|<\delta,|y|<\delta$ is defined as $V(f, S)=\sup _{\left[I_{v}\right]} \sum\left|\Delta\left(I_{v}, f\right)\right|$ where $\left[I_{v}\right]$ traverses all finite sets of nonoverlapping intervals in $S$. To establish $V(f, S) \leqq M$ it suffices to show $V\left(f, S^{\prime}\right)<M$ for every rectangle $S^{\prime}:|x|<\delta^{\prime}<\delta,|y|<\delta^{\prime \prime}<\delta$. If $V(f, S)$ is finite, $f(x, y)$ is called of bounded variation in $S$ in the sense of Lebesgue. According to a fundamental theorem of Lebesgue ${ }^{18}$ $V(f, S)<\infty$ implies that almost everywhere in $S$

16 Compare Busemann-Feller [1, p. 7].

${ }^{17}$ See Denjoy [1].

13 See Lebesgue [1] or Saks [1]. 


$$
\lim _{p \rightarrow \infty} \Delta\left(I_{p}, f\right) /\left|I_{p}\right|=F\left(x_{0}, y_{0}\right), \quad\left|I_{p}\right|=\text { area of } I_{p},
$$

exists and is finite, provided $\left(x_{0}, y_{0}\right) \in \bar{I}_{\nu}$ and the sequence $\left\{I_{\nu}\right\}$ is regular, that is, if $h_{\nu}$ and $k_{v}$ are the sides of $I_{v}$, then

$$
0<\lim \inf \frac{h_{\nu}}{k_{\nu}} \leqq \lim \sup \frac{h_{\nu}}{k_{\nu}}<\infty .
$$

4. The existence of the second differential. After these preparations we show first:

(4.1) If $f$ is defined and continuous for $|x| \leqq \delta,|y| \leqq \delta$ and if for some $\alpha, 0<\alpha<\pi / 2$, the slopes of the sections $x \sin \alpha \pm y \cos \alpha=$ const. of $z=f(x, y)$ have variation at most $B$, then $f(x, y)$ has in $S:|x|<\delta$, $|y|<\delta$ at most variation $2 \delta B / \cos \alpha$ and $z=f(x, y)$ has a tangent plane a.e., that is, the derivatives $f_{\beta}^{\prime}$ exist simultaneously for all $\beta$ and

$$
f_{\beta}^{\prime}=f_{0}^{\prime} \cos \beta+f_{\pi / 2}^{\prime} \sin \beta .
$$

Proof. For given $\delta^{\prime}, \delta^{\prime \prime}<\delta$ choose a rational number $r=p / q$ so that $\delta^{\prime \prime} \leqq r \delta^{\prime} \cdot \tan \alpha<\delta$. For any $N$ that is divisible by $q$ let $I_{\mu \nu}^{N}$ denote the interval $[(\mu-1) / N-1] \delta^{\prime}<x<(\mu / N-1) \delta,\{(\nu-1) / N-r\} \delta^{\prime} \tan \alpha$ $<y<(\nu / N-r) \delta^{\prime} \tan \alpha, \mu=1, \cdots, 2 N ; \nu=1, \cdots, 2 N r=N^{\prime}$. Then the diagonals of $I_{\mu \nu}^{N}$ are along lines $x \cdot \sin \alpha \pm y \cdot \cos \alpha=$ const. Since $f(x, y)$ is continuous it suffices to show that

$$
\sum_{\mu, v=1}^{2 N, N^{\prime}}\left|\Delta\left(I_{\mu \nu}^{N}, f\right)\right| \leqq 2 \delta^{\prime} B / \cos \alpha \text { for every } N \text { divisible by } q .
$$

Let $p_{\mu \nu}(s, t)$ denote the point $x=\delta^{\prime}[(2 \mu-1-2 N+s) /(2 N)], y$ $=\delta^{\prime} \tan \alpha[(2 \nu-1-2 r N+t) /(2 N)]$ so that $p_{\mu \nu}(0,0)$ is the center of $I_{\mu \nu}^{N}$. Then

$$
\begin{aligned}
\Delta\left(I_{\mu \nu}^{N}, f\right)= & \left\{\left[f\left(p_{\mu \nu}(1,1)\right)-f\left(p_{\mu \nu}(0,0)\right)\right]-\left[f\left(p_{\mu \nu}(0,0)\right)\right.\right. \\
& \left.\left.-f\left(p_{\mu \nu}(-1,-1)\right)\right]\right\}-\left\{\left[f\left(p_{\mu \nu}(1,-1)\right)-f\left(p_{\mu \nu}(0,0)\right)\right]\right. \\
& \left.-\left[f\left(p_{\mu \nu}(0,0)\right)-f\left(p_{\mu \nu}(-1,1)\right)\right]\right\} .
\end{aligned}
$$

Hence by (3.1)

$$
\begin{aligned}
\Delta\left(I_{\mu \nu}^{N}, f\right)= & \left(\delta^{\prime} /(2 N \cos \alpha)\right)\left\{f_{\alpha}^{*}\left(p_{\mu \nu}\left(\theta^{1}, \theta^{1}\right)\right)\right. \\
- & \left.f_{\alpha}^{*}\left(p_{\mu \nu}\left(-\theta^{2},-\theta^{2}\right)\right)\right\}-\left(\delta^{\prime} /(2 N \cos \alpha)\right) \\
& \cdot\left\{f_{-\alpha}^{*}\left(p_{\mu \nu}\left(\theta^{3},-\theta^{3}\right)\right)-f_{-\alpha}^{*}\left(p_{\mu \nu}\left(-\theta^{4}, \theta^{4}\right)\right)\right\},
\end{aligned}
$$


where $0<\theta^{i}<1$ and the $\theta^{i}$ depend on $\mu$ and $\nu$. Therefore

$$
\begin{aligned}
\sum_{\mu, \nu=1}^{2 N, N^{\prime}}\left|\Delta\left(I_{\mu \nu}^{N}, f\right)\right| \leqq & \left(\delta^{\prime} /(2 N \cos \alpha)\right) \sum_{\lambda=1}^{2 N} \sum_{\mu=\nu=\lambda} \mid f_{\alpha}^{*}\left(p_{\mu \nu}\left(\theta^{1}, \theta^{1}\right)\right) \\
& -f_{\alpha}^{*}\left(p_{\mu \nu}\left(-\theta^{2},-\theta^{2}\right)\right) \mid \\
& +\left(\delta^{\prime} /(2 N \cos \alpha)\right) \sum_{\lambda=1}^{N^{\prime}} \sum_{\mu+\nu=\lambda} \mid f_{\alpha}^{*}\left(p_{\mu \nu}\left(\theta^{8},-\theta^{3}\right)\right) \\
& -f_{\alpha}^{*}\left(p_{\mu \nu}\left(-\theta^{4}, \theta^{4}\right)\right) \mid .
\end{aligned}
$$

Since the variation of $f_{ \pm \alpha}^{*}$ along the lines $x \cdot \sin \alpha \pm y \cdot \cos \alpha=$ const. is at most $B$ and $\mu-\nu=\lambda$ implies that $p_{\mu \nu}\left(\theta^{1}, \theta^{1}\right)$ and $p_{\mu \nu}\left(-\theta^{2},-\theta^{2}\right)$ lie on the line $x \cdot \sin \alpha-y \cdot \cos \alpha=\delta \lambda-\sin \alpha-r \cdot \cos \alpha$ the relation (4.3) follows.

The derivative $f_{\alpha}^{\prime}(x, y)$ exists a.e. along a fixed line $x \cdot \sin \alpha$ $-y \cdot \cos \alpha=$ const. Since the two-dimensional set where $f_{\alpha}^{\prime}$ exists is measurable, it follows that $f_{\alpha}^{\prime}(x, y)$ exists a.e. in $|x| \leqq \delta,|y| \leqq \delta$. Denote by $N_{1}$ the set of measure 0 in $S$ where either $f_{\alpha}^{\prime}$ or $f_{-\alpha}^{\prime}$ or the set derivative $F(x, y)$ of $f(x, y)$ does not exist. If $\left(x_{0}, y_{0}\right) \in S-N_{1}$ and $k=h \cdot \cos \alpha, l=h \cdot \sin \alpha$, then

$$
\begin{aligned}
F\left(x_{0}, y_{0}\right)=\lim \frac{1}{k l}\left\{f \left(x_{0}+k, y_{0}\right.\right. & +l)+f\left(x_{0}, y_{0}\right) \\
& \left.-f\left(x_{0}+k, y\right)+f\left(x_{0}, y_{0}+l\right)\right\} \\
=\lim \frac{1}{k l}\left\{-f\left(x_{0}+\right.\right. & \left.k, y_{0}-l\right)-f\left(x_{0}, y_{0}\right) \\
& \left.+f\left(x_{0}+k, y\right)+f\left(x_{0}, y_{0}-l\right)\right\} .
\end{aligned}
$$

Hence

$$
\begin{aligned}
2 F\left(x_{0}, y_{0}\right)= & \lim (k \sin \alpha)^{-1}\left\{\frac{f\left(x_{0}+k, y_{0}+l\right)-f\left(x_{0}, y_{0}\right)}{h}\right. \\
& -\frac{f\left(x_{0}+k, y_{0}-l\right)-f\left(x_{0}, y_{0}\right)}{h} \\
& \left.-2 \sin \alpha \frac{f\left(x_{0}, y_{0}+l\right)-f\left(x_{0}, y_{0}-l\right)}{2 l}\right\} .
\end{aligned}
$$

The first two terms in the braces tend to $f_{\alpha}^{\prime}\left(x_{0}, y_{0}\right)$ and $f_{-\alpha}^{\prime}\left(x_{0}, y_{0}\right)$ respectively, therefore the last term converges, that is, $D_{x / 2}^{\prime} f\left(x_{0}, y_{0}\right)$ exists. Similarly $D_{0}^{\prime} f\left(x_{0}, y_{0}\right)$ exists. It follows from the last section that $f_{0}^{\prime}$ and $f_{\pi / 2}^{\prime}$ exist a.e. in $S-N_{1}$, so that $f_{0}^{\prime}, f_{\pi / 2}^{\prime}, f_{ \pm \alpha}^{\prime}$ and $F$ exist in $S$ except for a set $N_{2} \supset N_{1}$ of measure 0 . 
Let $\left(x_{0}, y_{0}\right) \in S-N_{2}, \quad 0<\beta<\pi, \beta \neq \pi / 2$, and put $h \cdot \cos \beta=k$, $h \cdot \sin \beta=l$. By transforming (4.4) it follows that

$$
\begin{aligned}
F\left(x_{0}, y_{0}\right)= & \lim (h \cdot \sin \beta \cos \beta)^{-1}\left\{\frac{1}{h}\left(f\left(x_{0}+k, y_{0}+l\right)-f\left(x_{0}, y_{0}\right)\right)\right. \\
& -\frac{\cos \beta}{k}\left(f\left(x_{0}+k, y_{0}\right)-f\left(x_{0}, y_{0}\right)\right) \\
& \left.-\frac{\sin \beta}{l}\left(f\left(x_{0}, y_{0}+l\right)-f\left(x_{0}, y_{0}\right)\right)\right\} .
\end{aligned}
$$

The last part in the braces tends to $-\cos \beta f_{0}^{\prime}-\sin \beta f_{\pi / 2}^{\prime}$, hence the first part converges and (4.2) follows.

This theorem leads to the following main result on functions $f(x, y)$ for the present paper.

(4.5) THEOREM. If $f$ is defined and continuous for $|x| \leqq \delta,|y| \leqq \delta$, and if the slopes of the sections $y=$ const. and $x \cdot \sin \alpha \pm y \cdot \cos \alpha=$ const., for some fixed $\alpha, 0<\alpha<\pi / 2$, have variation at most $B$, then the following facts hold simultaneously almost everywhere in $S(|x|<\delta,|y|<\delta)$.

(1) The set derivative $F(x, y)$ of $f(x, y)$ exists.

(2) $z=f(x, y)$ has a tangent plane.

(3) $d_{\beta}^{\prime \prime} f(x, y)$ exists for all $\beta$ in the following strong sense:

$$
\begin{aligned}
& d_{\beta}^{\prime \prime} f\left(x_{0}, y_{0}\right) \\
& =\lim _{\bar{\beta} \rightarrow \beta, h \rightarrow 0} \frac{2}{h}\left\{\frac{f\left(x_{0}+h \cdot \cos \bar{\beta}, y_{0}+h \cdot \sin \bar{\beta}\right)-f\left(x_{0}, y_{0}\right)}{h}-f_{\bar{\beta}}\left(x_{0}, y_{0}\right)\right\}
\end{aligned}
$$

and

$$
d_{\beta}^{\prime \prime} f=d_{0}^{\prime \prime} f \cos ^{2} \beta+2 F \cdot \cos \beta \sin \beta+d_{\pi / 2}^{\prime \prime} \sin ^{2} \beta .
$$

ProOF. By (4.1) and previous remarks the following statements are true except for a set $N_{3}$ of measure $0: f_{\beta}^{\prime}$ exists for all $\beta$ and $f_{\beta}^{\prime}=f_{0}^{\prime} \cos \beta+f_{\pi / 2}^{\prime} \sin \beta$; the derivatives $d_{0}^{\prime \prime} f$ and $d_{ \pm \alpha} f$ exist. It is important that in the first part of this proof only the existence of $d_{0}^{\prime \prime} f$ is used and not the fact that the slopes of $y=$ const. have variation at most $B$. Let $\left(x_{0}, y_{0}\right) \in S-N_{3}$. Put $f_{0}(s, t)=f\left(x_{0}+s, y_{0}+t\right)$, $f_{0}=f(0,0)=f\left(x_{0}, y_{0}\right)$ and $k=h \cdot \cos \alpha, l=h \cdot \sin \alpha$. Then

$$
\begin{aligned}
\sin \alpha \cos \alpha F\left(x_{0}, y_{0}\right)= & \lim h^{-2}\left\{f_{0}(k, l)+f_{0}-f_{0}(k, 0)-f(0, l)\right\} \\
= & \lim h^{-2}\left\{f_{0}+f_{0}(-k,-l)\right. \\
& \left.\quad-f_{0}(-k, 0)-f_{0}(0,-l)\right\},
\end{aligned}
$$


hence

$$
\begin{aligned}
2 \cdot \sin \alpha \cos \alpha F\left(x_{0}, y_{0}\right)= & \lim \left\{h^{-2}\left(f_{0}(k, l)+f_{0}(-k,-l)-2 f_{0}\right)\right. \\
& -k^{-2} \cos ^{2} \alpha\left(f_{0}(k, 0)+f_{0}(-k, 0)-2 f_{0}\right) \\
& \left.-l^{-2} \sin ^{2} \alpha\left(f_{0}(0, l)+f(0, l)-2 f_{0}\right)\right\} .
\end{aligned}
$$

The first two terms in the braces tend to $D_{\alpha}^{\prime} f=d_{\alpha}^{\prime \prime} f$ and $-\cos ^{2} \alpha D_{0}^{\prime \prime} f$ $=-\cos ^{2} \alpha d_{0}^{\prime \prime} f$, hence the last term converges, so that $D_{x / 2}^{\prime \prime} f\left(x_{0}, y_{0}\right)$ exists. By (3.2) the derivative $d_{\pi / 2}^{\prime \prime} f$ will exist except at the set $N_{4} \supset N_{3}$ of measure 0 .

Let $\left(x_{0}, y_{0}\right) \in S-N_{4}$ and $\bar{\beta} \rightarrow \beta^{*}$, then by (4.2)

$$
f_{\bar{\beta}}^{\prime}\left(x_{0}, y_{0}\right)=f_{0}^{\prime} \cos \bar{\beta}+f_{\pi / 2}^{\prime} \sin \bar{\beta} \rightarrow f_{\beta^{*}}^{\prime}\left(x_{0}, y_{0}\right) .
$$

If $\bar{k}=h \cdot \cos \bar{\beta}, \bar{l}=h \cdot \sin \bar{\beta}$, and $0<\beta^{*}<\pi, \beta^{*} \neq \pi / 2$, then the regularity condition (3.4) is satisfied for $h \rightarrow 0$ and $\bar{\beta} \rightarrow \beta^{*}$. Therefore

$$
\begin{aligned}
2 \sin \bar{\beta} \cos \bar{\beta} F\left(x_{0}, y_{0}\right)= & \lim _{\bar{\beta} \rightarrow \beta^{*}, h \rightarrow 0}\left\{\frac{2}{h}\left(\frac{f_{0}(\bar{k}, \bar{l})-f_{0}}{h}-f_{\bar{\beta}}^{\prime}\right)\right. \\
& -\frac{2 \cos ^{2} \bar{\beta}}{\bar{k}}\left(\frac{f_{0}(\bar{k}, 0)-f_{0}}{\bar{k}}-f_{0}^{\prime}\left(x_{0}, y_{0}\right)\right) \\
& \left.-\frac{2 \sin ^{2} \beta}{\bar{l}}\left(\frac{f_{0}(0, \bar{l})-f_{0}}{\bar{l}}-f_{x / 2}^{\prime}\left(x_{0}, y_{0}\right)\right)\right\} .
\end{aligned}
$$

The last part of the terms in braces tends to $d_{0}^{\prime \prime} f\left(x_{0}, y_{0}\right) \cos ^{2} \beta^{*}$ $+d_{\pi / 2} \sin ^{2} \beta^{*}$, respectively. Therefore the first part has a limit, which proves (4.7) for all $\beta^{*}$ and (4.6) for $\beta^{*} \neq 0, \pi / 2$. The proof fails for these two values because the regularity condition (3.4) does not hold.

However, we now apply our result to the coordinate system $\xi=x \cdot \sin \alpha / 2+y \cdot \cos \alpha / 2, \quad \eta=-x \cdot \cos \alpha / 2+y \cdot \sin \alpha / 2$. The lines $y$ $=$ const. and $x \cdot \sin \alpha+y \cdot \cos \alpha=$ const. have then the equations $\xi \cdot \sin \alpha / 2 \pm \eta \cdot \cos \alpha / 2=$ const. By hypothesis the slopes of these lines have variation at most $B$; moreover, it was proved that $d^{\prime \prime} f$ exists along the lines $\eta=$ const. As was pointed out in the beginning, the first part of the proof used only the existence a.e. of $d^{\prime \prime} f$ along $y=$ const., hence it follows that (4.6) holds for all directions except those parallel to the $\xi$-or $\eta$-axis; (4.6) holds therefore in particular in the direction of the old $x$ - and $y$-axes. This completes the proof of (4.5). A simple application of (4.6) and (4.7) yields

(4.8) CoROLLARY. Under the assumptions of (4.5) the function $f(x, y)$ has almost everywhere a second differential in the sense of Stolz, that is, the relation 


$$
\begin{aligned}
f\left(x_{0}+h, y_{0}+k\right)= & f\left(x_{0}, y_{0}\right)+h f_{0}^{\prime}(x, y)+k f_{\pi / 2}^{\prime} \\
& +\left(h^{2} d_{0}^{\prime \prime} f+2 h \cdot k \cdot F+k^{2} d_{\pi / 2}^{\prime \prime} f\right) / 2+o\left(h^{2}+k^{2}\right)
\end{aligned}
$$

holds for almost all $x_{0}, y_{0}$.

Relation (4.9) implies that

$$
\begin{aligned}
F(x, y)=\lim _{h \rightarrow 0, k \rightarrow 0}\left\{f\left(x_{0}+h, y_{0}+k\right)\right. & +f\left(x_{0}, y_{0}\right) \\
& \left.-f\left(x_{0}+h, y_{0}\right)-f\left(x_{0}, y_{0}+k\right)\right\}
\end{aligned}
$$

without any regularity condition. If $f_{0}^{\prime}$ and $f_{\pi / 2}^{\prime}$ exist in a neighborhood of $\left(x_{0}, y_{0}\right)$ it follows that the mixed partial derivatives $\partial^{2} f / \partial x \cdot \partial y$ and $\partial^{2} f / \partial y \cdot \partial x$ exist at $\left(x_{0}, y_{0}\right)$ and equal $F\left(x_{0}, y_{0}\right)$. A similar interpretation can be given to $F\left(x_{0}, y_{0}\right)$ in the general case, however this question is alien to the geometric purpose of the present paper.

The theorem of Euler on the curvatures of the normal sections of $z=f(x, y)$ at $\left(x_{0}, y_{0}\right)$ follows from (4.2) and (4.7) alone. ${ }^{19}$ In the present case both the theorems of Euler and of Meusnier follow immediately from (4.9). For it follows from II of $\$ 3$ that the paratingens at $\left(x_{0}, y_{0}\right)$ does not contain the normal to the tangent plane $I I$ of $z=f(x, y)$ at $\left(x_{0}, y_{0}\right)$. If $(\bar{x}, \bar{y}, \bar{z})$ are new coordinates with $\Pi$ as $(\bar{x}, \bar{y})$-plane and $\left(x_{0}, y_{0}\right)$ as origin, then $z=f(x, y)$ will in a neighborhood of $(0,0)=\left(x_{0}, y_{0}\right)$ be represented in the form $\bar{z}=\bar{f}(\bar{x}, \bar{y})$. Then $o\left(\left(x-x_{0}\right)^{2}+\left(y-y_{0}\right)^{2}\right)=o\left(\bar{x}^{2}+\bar{y}^{2}\right)$. The relation (4.9) is equivalent to the statement that $f(x, y)$ coincides, in a neighborhood of $\left(x_{0}, y_{0}\right)$, except for $o\left(\left(x-x_{0}\right)^{2}+\left(y-y_{0}\right)^{2}\right)$, with a paraboloid $a_{1}+a_{2} x+a_{3} y+a_{4} x^{2}$ $+a_{6} x y+a_{6} y^{2}$. This same paraboloid written in the new coordinates will approximate $\bar{z}=f(\bar{x}, \bar{y})$ up to $o\left(\left(x-x_{0}\right)^{2}+\left(y-y_{0}\right)^{2}\right)=o\left(\bar{x}^{2}+\bar{y}^{2}\right)$. In the new coordinates $(4.9)$ reads as

$$
\bar{f}(\bar{x}, \bar{y})=\left(\bar{x}^{2} d_{0}^{\prime \prime} \bar{f}+2 \cdot \bar{x} \cdot \bar{y} \cdot \bar{F}+\bar{y}^{2} d_{\pi / 2}^{\prime \prime} \bar{f}\right) / 2+o\left(\bar{x}^{2}+\bar{y}^{2}\right)
$$

from which it follows that

$$
d_{\beta}^{\prime \prime} \bar{f}(0,0)=\cos ^{2} \beta d_{0}^{\prime \prime} \bar{f}+2 \cdot \cos \beta \sin \beta \bar{F}+\sin ^{2} \beta d_{\pi / 2}^{\prime \prime} \bar{f} .
$$

But now $d_{\beta}^{\prime \prime} f(0,0)$ is the curvature of the section $\bar{x} \cdot \sin \beta+\bar{y} \cdot \cos \beta=0$ with $\bar{z}=f(\bar{x}, \bar{y})$, so that (4.10) is Euler's theorem.

Meusnier's theorem can easily be derived from $\left(4.9^{\prime}\right)$ in the following form (cf. Bouligand $[1$, p. 120]): Suppose that the surface $z=f(x, y)$ has a second differential in the sense of Stolz (cf. (4.9)) at $P=\left(x_{0}, y_{0}, f\left(x_{0}, y_{0}\right)\right)$. Then any two curves on the surface with the

${ }^{19}$ Compare Busemann-Feller [1, pp. 28-29]. 
same tangent and same osculating plane at $P$ have there the same curvature; and the circles of curvature of all plane sections with a given tangent at $P$ lie on a sphere.

We apply these results to the neighborhood $\left|x^{\prime}\right| \leqq \eta,\left|y^{\prime}\right| \leqq \eta$ of (a), (b), (c) of $\S 3$. Because of (b) the Lebesgue area of the surface $z^{\prime}=f\left(x^{\prime}, y^{\prime}\right)$ over $\left|x^{\prime}\right| \leqq \eta,\left|y^{\prime}\right| \leqq \eta$ is finite; it equals the area of the corresponding piece $\bar{\Delta}$ of the original surface $\Phi$, where $\bar{\Delta}$ is the closure of the open set $\Delta$ in the $(\mu, \nu)$-plane corresponding to $\left|x^{\prime}\right|<\eta$, $\left|y^{\prime}\right|<\eta$. In order to avoid the known difficulties regarding the additivity of area, observe that $\Phi$ is, according to Lindelöf's theorem, covered by a countable number of sets $\bar{\Delta}$. This yields the result of the introduction, which may be stated more precisely as follows:

(4.11) THEOREM. If the surface $\Phi$ satisfies the hypotheses I, II, III of $\S 3$, then $\Phi$ is the union of a countable number of pieces $\bar{\Delta}$ of finite area, such that for almost all points $q$ of $\bar{\Delta}$ the following statements hold simultaneously: $\Phi$ has a tangent plane $\Pi$ at $q$. All curves on $\bar{\Delta}$ through $q$ which have an osculating plane, but different from $\pi$, have a curvature at $q$; and these curvatures satisfy the theorems of Euler and Meusnier.

\section{A. Alexandrov}

\section{REFERENCES}

1. Almost everywhere existence of second differentials of convex functions and some properties of convex manifolds connected with it (Russian), Leningrad State Uni-

G. Bouligand versity Annals (Uchenye Zapiski), Mathematics Series, vol. 6 (1939) pp. 3-35.

1. Introduction à la géometrie infinitésimale directe, Vuibert, Paris, 1932.

H. BusemanN aNd W. Feller

1. Krümmungseigenschaften konvexer Flächen, Acta Math. vol. 66 (1935) pp. 1-47.

A. Denjoy

1. Sur l'intégration des coefficientes différentiels d'ordre supérieur, Fund. Math. vol. 25 (1935) pp. 273-326.

O. HAUPT

1. Ueber die Krïmmung ebener Bogen endlicher Ordnung, Sitzungsberichte der physikalisch-medicinischen Societät zu Erlangen vol. 71 (1939) pp. 219-227.

J. HJELMSLEV

1. Contribution d la géometrie infinitésimale de la courbe réele, Oversigt over det Kongelige Danske Videnskabernes Selskabs Forhandlinger, 1911, pp. 433-454.

2. Die graphische Geometrie, Comptes Rendus du Huitième Congrès des Mathématicians Scandinaves, Lund, 1935, pp. 2-12.

B. JESSEN

1. Om konvekse Kurvers Krumming, Mathematisk Tidsskrift B, 1929, pp. 50-62.

H. LEBESGUE

1. Sur l'intêgration des fonctions discontinues, Ann. Ecole Norm. (3) vol. 27 (1910) pp. 361-450.

A. MARChaud

1. Sur les continus d'ordre borné, Acta Math. vol. 55 (1930) pp. 67-115. 
J. Marcinkiewicz and A. Zygmund

1. On the differentiability of functions and summability of trigonometrical series, Fund. Math. vol. 26 (1936) pp. 1-43.

S. SAKS

1. Theory of the integral, Monografie Matematyczne, vol. 7, Warsaw and New York (Stechert), 1937.

ILLINOIS INSTITUTE OF TECHNOLOGY AND BROWN UNIVERSITY

\section{INTEGRAL DISTANCES}

NORMAN H. ANNING AND PAUL ERDÖS

In the present note we are going to prove the following result:

For any $n$ we can find $n$ points in the plane not all on a line such that their distances are all integral, but it is impossible to find infinitely many points with integral distances (not all on a line). ${ }^{1}$

Proof. Consider the circle of diameter $1, x^{2}+y^{2}=1 / 4$. Let $p_{1}, p_{2}, \cdots$ be the sequence of primes of the form $4 k+1$. It is well known that

$$
p_{i}^{2}=a_{i}^{2}+b_{i}^{2}, \quad a_{i} \neq 0, \quad b_{i} \neq 0,
$$

is solvable. Consider the point (on the circle $x^{2}+y^{2}=1 / 4$ ) whose distance from $(-1 / 2,0)$ is $b_{i} / p_{i}$. Denote this point by $\left(x_{i}, y_{i}\right)$. Consider the sequence of points $(-1 / 2,0),(1 / 2,0),\left(x_{i}, y_{i}\right), i=1,2, \ldots$ We shall show that any two distances are rational. Suppose this has been shown for all $i<j$. We then prove that the distance from $\left(x_{j}, y_{j}\right)$ to $\left(x_{i}, y_{i}\right)$ is rational. Consider the 4 concyclic points $(-1 / 2,0),(1 / 2,0)$, $\left(x_{i}, y_{i}\right),\left(x_{j}, y_{j}\right) ; 5$ distances are clearly rational, and then by Ptolemy's theorem the distance from $\left(x_{i}, y_{i}\right)$ to $\left(x_{j}, y_{j}\right)$ is also rational. This completes the proof. Thus of course by enlarging the radius of the circle we can obtain $n$ points with integral distances.

It is very likely that these points are dense in the circle $x^{2}+y^{2}=1 / 4$, but this we can not prove. It is easy to obtain a set which is dense on $x^{2}+y^{2}=1 / 4$ such that all the distances are rational. Consider the

Received by the editors February 20, 1945.

${ }_{1}^{1}$ Anning gave 24 points on a circle with integral distances. Amer. Math. Monthly vol. 22 (1915) p. 321. Recently several authors considered this question in the Mathematical Gazette. 